\title{
Structural Architectures for a Deployable Wideband UHF Antenna
}

\author{
Gina M. Olson ${ }^{1}$ and Sergio Pellegrino ${ }^{2}$ \\ California Institute of Technology, Pasadena, CA, 91125 \\ Joseph Costantine ${ }^{3}$ \\ California State University - Fullerton, Fullerton, CA, 92834 \\ and \\ Jeremy Banik ${ }^{4}$ \\ Air Force Research Laboratory, Space Vehicles Directorate, Kirtland Air Force Base, New Mexico, 87117
}

This paper explores concepts for a wideband deployable antenna suitable for small satellites. The general approach taken was to closely couple antenna theory and structural mechanics, to produce a deployable antenna that is considered efficient in both fields. Approaches that can be deployed using stored elastic strain energy have been favored over those that require powered actuators or environmental effects to enforce deployment. Two types of concepts were developed: thin shell structure and pantograph. These concepts cover four antenna topologies: crossed log periodic, conical log spiral, helical and quadrifilar helix. Of these, the conical log spiral antenna and the accompanying deployment concepts are determined to be the most promising approaches that warrant further study.

\section{Introduction}

A NTENNAS are required for all wireless communication and wireless data transfer, both terrestrial and in outer space. A high gain, wideband deployable antenna suitable for use on small satellites could enable capabilities typically found on much larger satellites. For example, a compact antenna-satellite system could be used as a communications relay station. Narrowband antennas have limited effectiveness for this application; on the other hand wider band antennas are more versatile and allow faster data transfer.

One popular small satellite platform is the CubeSat. A 1U CubeSat has maximum dimensions of $10 \mathrm{~cm} \times 10 \mathrm{~cm}$ $\times 10 \mathrm{~cm}$. Three of these cubes are commonly joined in a stack to form a $3 \mathrm{U}$ CubeSat. The CubeSat platform currently attracts interest from many universities, companies and government organizations for its advertised low cost and quick access to space. The current predominant antenna choice for university CubeSats is one or more dipole (or monopole) type antennas [1]. Dipole antennas are omnidirectional and if correctly arranged can mimic circular polarization, a desired quality that limits the required pointing control. Furthermore, dipoles are straightforward to design and deploy, and designs specific to CubeSats are available commercially. Examples of CubeSats that have flown with dipole antennas include Tokyo Institute of Technology's Cute 1.7+ APD II and California Polytechnic's CP1 (destroyed in launch failure) [2,3].

Despite their popularity, dipoles are not the optimal solution in many cases since the design is generally narrowband, low gain and inefficient [1]. A different antenna type is required to achieve a significant increase in bandwidth. Prior work into small satellite antennas has suggested patch antennas (which do not have to be deployed) and a deployable hemispherical helical antenna that flattens into a single plane $[4,1]$. Further, active antennas

\footnotetext{
${ }^{1}$ Graduate Student, Graduate Aerospace Laboratories, 1200 E. California Blvd, MC 205-45, AIAA Student Member, golson@caltech.edu

2 Joyce and Kent Kresa Professor of Aeronautics and Professor of Civil Engineering, Graduate Aerospace Laboratories, 1200 E. California Blvd, MC 301-46, AIAA Fellow, sergiop@ caltech.edu

3 Assistant Professor, Electrical Engineering Department, $800 \mathrm{~N}$ State College Blvd, E-100A jcostantine@fullerton.edu

${ }^{4}$ Research Mechanical Engineer, 3550 Aberdeen Ave SE, AIAA Senior Member.
} 
(which do not have to be deployed) have been considered by the University of Hawaii for one of its own CubeSats [5]. Finally, a significant body of work exists on deployable parabolic reflectors which may be used in combination with a feed horn element to form a parabolic dish antenna.

Larger satellites use a variety of antenna types, each according to their unique frequency range requirements. Reflector style antennas are popular and horn antennas, which are highly directive, are useful for applications requiring global coverage [6]. In addition, helical antennas are used on many US satellites, including but not limited to Comsat, Fleetsat and Navstar-GPS [7]. Larger deployable satellite antennas have also been investigated by Lockheed Martin, the Harris Corporation and Astro Aerospace. Each have flown high gain mesh reflector antennas with deployed diameters in excess of $10 \mathrm{~m}$ [8-10]. Furthermore, Boeing recently deployed a reflector antenna with a diameter of $22 \mathrm{~m}$ [11]. Note, however, that these designs are complex and expensive and thus difficult to scale down to the size and cost required for most small satellites, including CubeSats. Additional concepts that might provide more inspiration are the designs for the UHF helical antenna on Skynet [12] and spring back reflectors [13].

Often the design of the radiating elements of an antenna and the design of an antenna's deployment structure are considered independently until late in the design process. Initial antenna geometry is selected to maximize antenna performance only, with little or no attention to structural efficiency or foldability. The study discussed here attempts to link the design of radiating elements and support structure early in the design process by formulating a conceptual design that is efficient as both an antenna and a deployable structure. This process begins by identifying the basic geometry of suitable antenna types and determining appropriate structural architectures to support and deploy those shapes. The antenna and structure must then be sized together since the structure itself affects antenna performance. This is an integrated process that frequently crosses back and forth between the principles of electromagnetic radiation and the principles of structural mechanics.

This paper uses this approach to develop new concepts for deployable wideband antennas suitable for small satellites, such as CubeSats. Limited pointing control is assumed so that directional antennas may be considered. The desired operating frequency range is $250-500 \mathrm{MHz}$, which covers part of both the Very High Frequency (VHF) and Ultra High Frequency (UHF) bands. Concepts should be lightweight, structurally simple and robust, and able to reliably achieve their deployed configuration.

\section{Antenna Design Requirements}

The fundamental function of antennas is to provide the link between electromagnetic waves and electrical current. The geometry of the conductive elements in the antenna (e.g. physical size, layout) and the material used determines the current distribution. This in turn determines most of the performance characteristics of the antenna such as the operating frequencies (where a large range constitutes a wideband antenna), radiation pattern and polarization. Within a specific antenna type, such as a dipole, the bandwidth can be modified slightly by changing the geometry, but large bandwidth changes require a different antenna type, such as a log periodic. Note that physically longer conductors resulting in larger (wider and taller) antennas are needed to receive lower frequencies.

The radiation pattern of an antenna is a description of where the radiated power is sent and at what intensity. Highly directive antennas focus the transmitted power into a narrow beam, ultimately achieving high gain at the penalty of requiring precise pointing.

Polarization is the pattern traced by the propagating electric field, and may be characterized as linear, circular or elliptical. Circular polarization is generally regarded as advantageous for satellites, particularly those with limited pointing control. This is because there is no signal loss due to rotation between circularly polarized transmitters and receivers. Circular polarization is required for the antenna studied here, and it was discovered to be the most restrictive constraint when evaluating acceptable antenna topologies. However, several techniques exist to make naturally linearly polarized antennas circularly polarized. For instance, though dipoles are linearly polarized, circular polarization can be mimicked by crossing two linearly polarized dipoles at an angle of $90^{\circ}$.

Two more design aspects must be considered. First, only the intended antenna elements should be made of conductive materials. Supporting elements should be made of electrically insulated materials; this places additional constraints on the problem studied here. Second, the deployment structures should use elastic strain energy to enforce deployment, since this approach is simple, reliable and low mass. Deployment actuators, cables, dampers and mechanical hinges are commonly used on large deployable structures, but these elements increase system complexity and cost and are therefore not appropriate for the small satellite application examined here.

\section{Antenna Structure Concepts}

To begin the process of identifying antenna types suitable to the case examined here, a comprehensive list of antenna designs was formed. The list was then reduced by eliminating antenna designs with similar topologies. The 
list was then further reduced to include only those antennas that can operate over the desired frequency range and be circularly polarized for some set of dimensions. The following antenna types were selected for further consideration: log periodic, reflector (dish), horn, helical, quadrifilar helix, conical log spiral, Lindenblad and patch. Note that the log periodic antenna must be composed of crossed dipole elements to achieve circular polarization.

Many structural concepts were considered, but only two basic types are presented below. The first is best characterized as a shell structure, while the second is best characterized as a pantograph. These two approaches cover four different antenna topologies that were selected based on best antenna performance potential (e.g. bandwidth, gain) and best deployment performance. The antenna topologies selected are log periodic, conical log spiral, helical and quadrifilar helix. All four exhibit high gain, circular polarization and wide bandwidth; in particular, the conical log spiral and log periodic antennas exhibit excellent bandwidth.

Packing any one of these antennas, however, presents significant challenges because the volume of the deployed antenna may be many times larger than the volume of the small satellite it must fit within. Consider, for instance, a helical antenna packaged into a $1 \mathrm{U}$ CubeSat. To receive a frequency of $250 \mathrm{MHz}$, such an antenna would occupy a cylindrical volume approximately $60 \mathrm{~cm}$ in diameter and approximately $100 \mathrm{~cm}$ tall, depending on the desired gain. To fit within the $1 \mathrm{U}$ CubeSat, a volumetric packaging ratio of 1:270 would be required. Similar or slightly lower ratios would be required for the other antenna topologies.

\section{A. Shell with Internal Elements}

The core idea of the shell structure concept is to form an ultra-thin composite shell in the shape of the deployed antenna with the conductive elements embedded within or attached to the laminate. The shell would be folded elastically and deployed using stored elastic strain energy. While it might be possible to fold appropriately shaped conductors without a shell, the shell helps to prevent snagging and provides structural support to the conductors, additional hold points and strain energy for deployment.

There are two different implementations of this concept. The first accommodates antennas that require conductors to be internal to the shell, such as a $\log$ periodic as shown in Figure 1. The second accommodates antennas in which the conductors lie on the perimeter of the shell, such as the helical, quadrifilar helix or conical log spiral antennas.

The log periodic antenna is more properly defined as an antenna array since it is composed of multiple dipoles of varying lengths. Each dipole is a

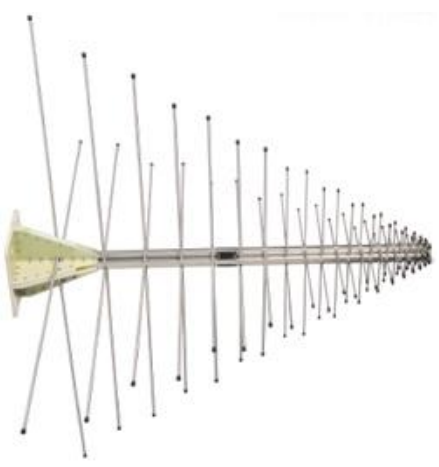

Figure 1. Commercially available crossed log periodic antenna [14].

narrowband antenna, and by combining the dipoles with a spacing that allows their frequencies to overlap, the collective antenna pattern is broadband. However, coupling between the conductive elements must be carefully controlled. While dipole antennas are omnidirectional, the log periodic antenna is directive and radiates out the small end of the antenna. The length of the longest element is determined by the lowest desired frequency, in this case $250 \mathrm{MHz}$, while the shortest element is determined by the highest frequency it must receive, in this case 500 MHz. The relationship between element spacing s, element length $l$ and element diameter $D$ for an ideal case is given by

$$
\frac{l_{n+1}}{l_{n}}=\frac{D_{n+1}}{D_{n}}=\frac{s_{n+1}}{s_{n}}
$$

while the length $l$ of a half-wave dipole intended to receive a frequency $f$ in free space is most commonly estimated by

$$
l=\frac{\lambda}{2}=\frac{c}{2 f}
$$

where $\lambda$ is the wavelength and $c$ is the speed of light.

The crossed log periodic antenna fits neatly into either a square pyramid or a cone. Consider a square pyramid of base length $b$, base dipole $L$ and height $h$, where $L$ is also the length of the longest element in the antenna. A crossed $\log$ periodic antenna may be suspended within it by attaching the end of each conductive element to a corner of the pyramid, so the antenna lies along the diagonals. The shell could then provide the primary structural support and dimensional stability for the antenna. A possible method to fold this structure is shown in Figures 2 and 3. Note that this method requires a revolute joint at each crossing of the conducting arms. In Figure 2 the pyramid is flattened by first creasing two bisecting adjacent isosceles faces of the pyramid. 

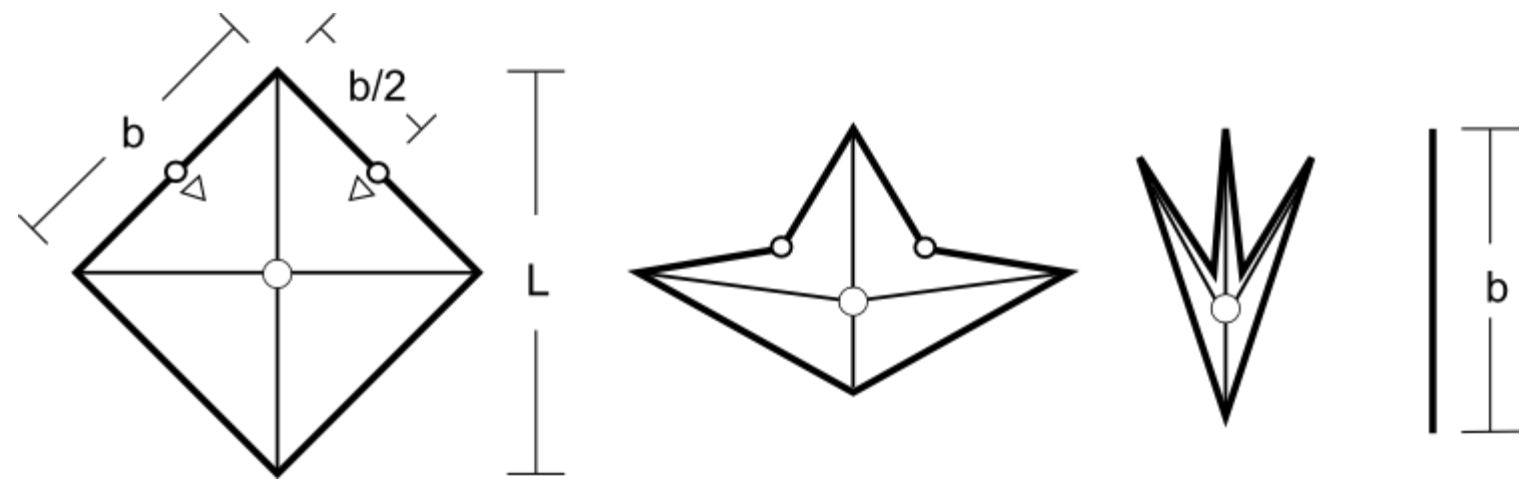

Figure 2. Flattening of log-periodic antenna, viewed from the base of the pyramid.

It is clear from Figure 2 that base length $b$ is $L / \sqrt{2}$. Note also that this method of flattening requires all conductive elements to shorten. The ratio between their deployed and folded length is constant along the height of the pyramid, and, since the longest element shortens from $L$ to $b$, the ratio is

$$
\beta=\frac{\sqrt{2}}{2}
$$

There are many ways to accommodate this shortening. The two proposed here are telescoping rods and compliant wires. Telescopic rods have been used extensively in the antenna industry, mostly in dipole or monopole applications such as a portable radio antenna. Only one telescoping stage would be required here since the ratio of the folded to deployed length is over 0.5. Compliant conductors could also be used, such as wires of a diameter small enough to allow elastic deformation or wire cages or flexible tubes with a conductive coating. Note that the conductors do not have to be circular; tall and thin rectangular conductors may also be used.

This same folding process is shown edge-on in Figure 3; the middle image shows the pyramid structure at the end of Figure 2.The structure may be additionally compacted by folding the flattened form in half, taking advantage of the revolute joints between antenna elements. Note that the antenna elements end before the vertex of the pyramid in Figure 3. The point in the pyramid at which the elements end is determined by the desired set of frequencies - the higher the frequency, the shorter the top elements.
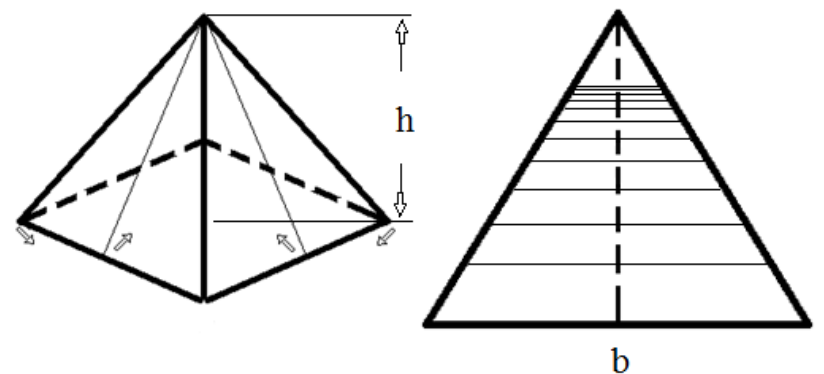

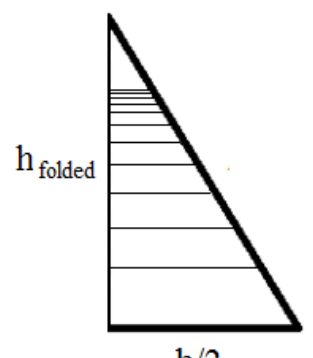

$\mathrm{b} / 2$

Figure 3. Face on folding steps as applied to a pyramid.

During the folding process shown in Figure 3, the pyramid height increases slightly as the pyramid folds behind one face. The final width is $b / 2=\sqrt{2} L / 4$, and the final height is

$$
h_{\text {folded }}=\sqrt{h^{2}+\frac{1}{8} L^{2}}
$$

Without details of the antenna or shell material, it is difficult to accurately estimate the stowed thickness of this antenna concept. Assuming very thin conductors and a solid shell, the thickness of the stowed configuration is estimated to be four times the minimum bending diameter of the selected shell material. 
Consider again the specific case of an antenna intended to receive frequencies between 250 and $500 \mathrm{MHz}$. Assume that the antenna must fit within a $1 \mathrm{U}$ CubeSat. Assuming a pure copper conductor with a circular cross section, the lowest conductor diameter was estimated to be $4 \mathrm{~mm}$. Hence, telescopic conductive elements are likely the simplest way to decrease element length.

The height is estimated to be $55 \mathrm{~cm}$, and the length of the longest element is estimated to be $60 \mathrm{~cm}$. The stowed height is $58.9 \mathrm{~cm}$, and the stowed width is $21.2 \mathrm{~cm}$. Thus, this concept does not compact enough to fit into a $1 \mathrm{U}$ CubeSat. The height could be sufficiently reduced by means of additional folds along horizontal lines between conductive elements, but introducing multiple crossing folds in the shell may be problematic.

Overall, deployable antenna concepts with internal conductive elements are more difficult to fold efficiently than the shells with perimeter elements. Depending on implementation, much of the external shell structure is not load bearing, and it may interfere with antenna performance. Specifically, it could reduce gain as the signal must travel through the shell.

\section{B. Shell with Perimeter Elements}

Next, the deployable shell architecture with perimeter elements is explored. Circular and square cross-section conductors cannot achieve as small a fold radius as thin rectangular conductors for a given cross-sectional area. Therefore only thin, wide conductors are considered here. In most cases, rectangular conductors can be substituted for circular with no loss in antenna performance.

Consider antenna elements that are either laminated or externally attached to the shell structure. This architecture applies to the conical log spiral, helical and quadrifilar helix antennas. Both the helical and quadrifilar helix antennas are cylindrical. The conical log spiral antenna, as the name suggests, fits a cone as illustrated by the commercially available version shown in Figure 4. Though this version has only one spiral, versions with two or even four spirals are also available. The shell architecture is demonstrated with the conical log spiral antenna.

Similar to the crossed log periodic concept, the first folding step with this concept is to flatten the shell. Note that in this case no special flattening method is used. The shell is simply flattened by introducing two diametrically opposite creases. After flattening, there are a number of ways the shell may be folded. The log periodic example demonstrated the need for extreme compaction ratios, suggesting many folds are needed. However, the shell must still be straightforward to deploy. To suit both of these requirements, the Miura-ori map fold pattern was selected to fold the shell the rest of the way. This fold pattern uses slanted fold lines to couple lateral and transverse compaction so the flattened shape can be unfolded by pulling on two diagonally opposite corners [16]. The folding steps are shown in Figure 5. The fold lines are shown in blue, representing mountain folds, and green, representing valley folds. An example conductor is shown in yellow on the first and second images.
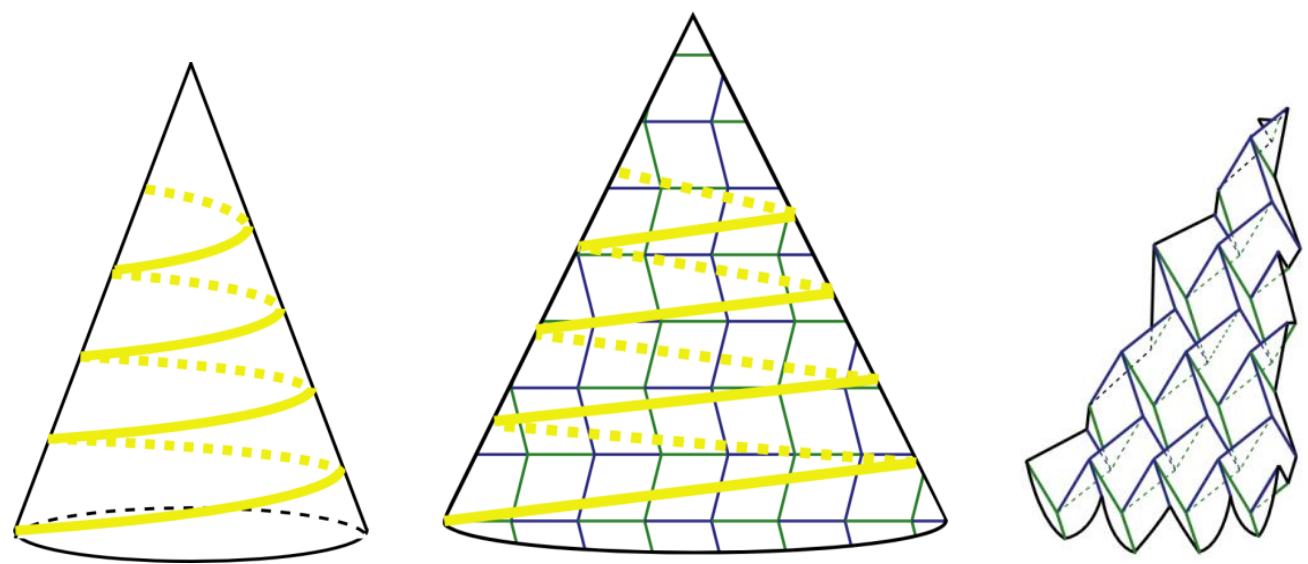

Figure 5. Conical log spiral antenna folded using Miura-Ori. 
The folded dimensions of this concept depend on the cell size and slant angle selected when applying the MiuraOri pattern. Within some limits, cell size and slant angle can be selected to fit within the desired dimensions. However, the smaller the cell size the greater the stowed thickness. The folds should be elastic to ensure deployment off stored strain energy is possible. Tight packaging may be achieved by forming a series of integral hinge lines in the shell structure where a softer matrix material (such as silicone) is used in the composite. Note that again the conductor terminates before the tip of the structure as determined by the highest desired frequency.

Implementations of this concept for the helical and quadrifilar helix are similar. The flattened shape is square so the Miura-ori pattern is similar to traditional, simpler applications.

\section{Helical Pantograph}

The next structural architecture is an extension of the basic linear pantograph, a sample of which is shown in Figure 6. A linear pantograph is made up of a series of interconnected scissor elements, each with the same angle. Changing the angle of one hinge changes the angle of all and thus changes the overall length of the structure.

This pantograph architecture is applicable to helical antennas. A commercially available helical antenna is shown in Figure 7. This structure is formed by nonstructural helically-shaped conductors connected by joints to one or more helical support rods.

The straight elements that make up a linear pantograph are replaced here by helices. The structure may be compacted axially much as the linear pantograph was - by decreasing the angle of connected elements (this also decreases the helix angle). However, this does not enforce compaction in the radial direction; it actually increases the radius slightly. To compact radially, the conductors must be folded elastically into a four leaf clover shape. Figure 8 illustrates this compaction process: the leftmost image is the deployed shape, the center image is the structure partway through axial compaction and the rightmost image is after radial compaction. The conductor (gold) is attached to two supporting helical rods (blue and red). Note that the scale is not the same between the second and third images, as this image is intended only to assist understanding of the concept. Also note that while the figure might seem to imply that only simple revolute joints are required, more degrees of
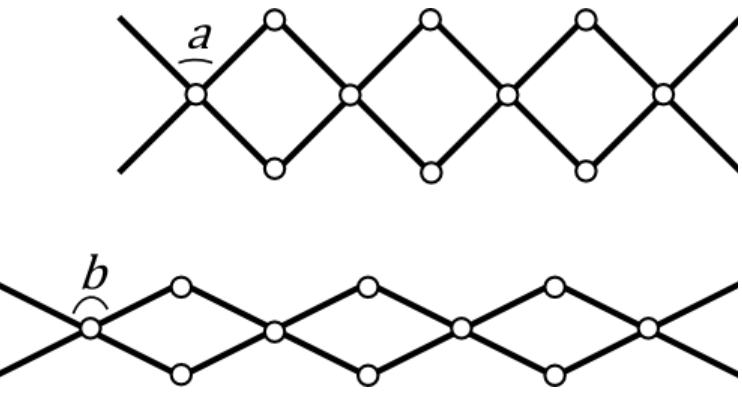

Figure 6. Linear pantograph example. freedom are likely needed.
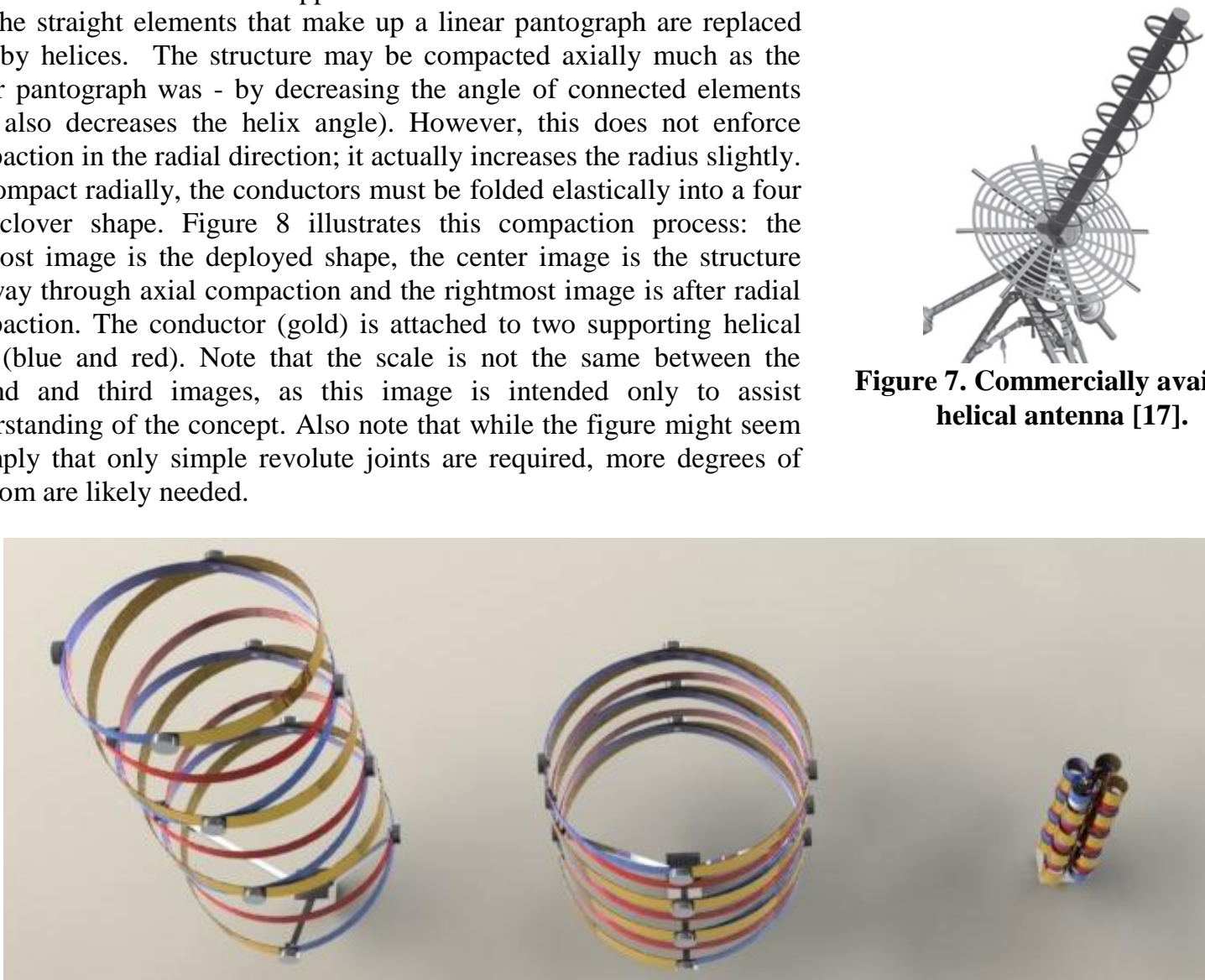

Figure 7. Commercially available helical antenna [17].

Figure 8. Helical pantograph concept shown fully deployed, axially compacted, and radially compacted. 
For large diameter antennas (required for receiving low frequencies) and very small satellites, such as CubeSats, this approach may not package to a sufficiently small volume. A simple extension of the above is simply to use eight leaves instead of four. Figure 9 shows a top down view of the four and eight leaf patterns modeled as twodimensional circles that fit inside a square.
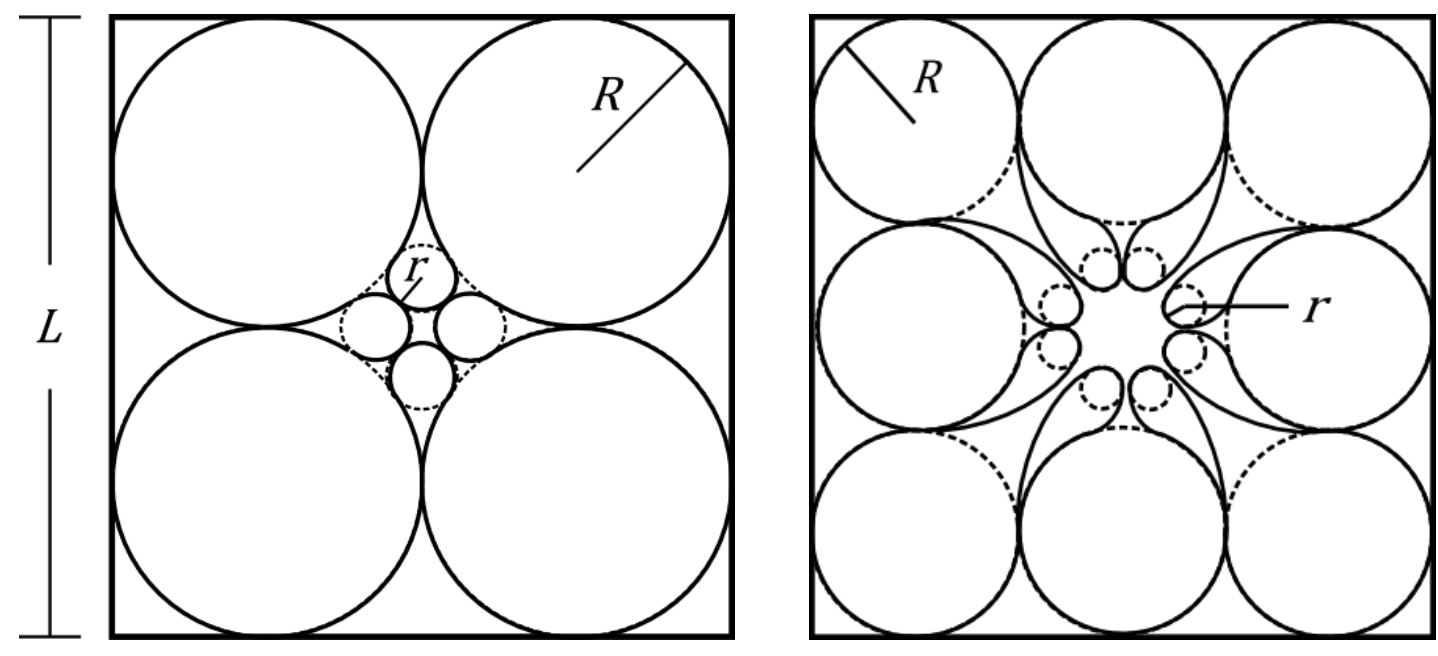

Figure 9. Two schemes for radial compaction of axially compacted helical pantograph.

Using these figures it is possible to estimate the maximum circumference that will fit within a square of side length $L$. A simple approach is to consider only the perimeter of the larger circles. In this case, the maximum circumference $C$ is

$$
\begin{aligned}
& C_{4}=2 \pi L \\
& C_{8}=\frac{8}{3} \pi L
\end{aligned}
$$

These estimates suggest there is a $\sim 25 \%$ advantage in moving to the second pattern. However, note that using only the large circles in the circumference estimate penalizes the second pattern more than the first. A better estimate would be

$$
C_{8}=\left(\frac{8}{3} \pi+\frac{4}{3}\right) L
$$

which includes the path to and from the center for the corner circles.

The minimum stowed height is set by the height of the conductors, and can be estimated with

$$
h_{\text {folded }}=(\# \text { turns })\left(h_{\text {conductor }}\right)
$$

and the minimum stowed cross sectional area is equal to the cross sectional area of the conductors and joints. However, the material strength will place limitations on the compaction since elastic deformation is desired. The conductors and support rods are sheared during axial compaction by a distance approximately equal to the vertical separation between turns of the helix. During radial compaction, the minimum elastic bend radius for the conductor (which depends on its thickness) may prevent the paths presented above from being reached.

Again consider the approximate dimensions of a helical antenna sized to receive the proper frequency range, 60 $\mathrm{cm}$ diameter and $1 \mathrm{~m}$ tall. Examining radial compaction, the four leaf clover pattern gives a packaged square with a side length of about $30 \mathrm{~cm}$, while the eight leaf pattern allows a side length of about $19 \mathrm{~cm}$. A more aggressive pattern would decrease these side lengths even further.

Finally, note that though this helical pantograph architecture applies most naturally to helical antennas, it may also be possible to adapt it to the quadrifilar helix and to the conical log spiral antennas. 


\section{Cylindrical Pantograph with Tension Elements}

While the helical pantograph concept offers high compaction ratios, it requires two folding steps. A second pantograph architecture is presented that applies to both helical and quadrifilar helix antennas. An example helical antenna was presented in Figure 7; a sample quadrifilar helix antenna is shown in Figure 10. The shape is similar to the helical antenna, but there are four conducting helices. This concept folds in only one step, and compacts in three directions simultaneously. The concept is an extension of circular pantographs; Figure 11 shows an example presented by You and Pellegrino in [19].

These pantographs are constructed of angulated instead of straight elements. Figure 12 shows an example of these elements.

A cylindrical pantograph that compresses in three perpendicular directions simultaneously may be constructed by attaching linear pantographs perpendicular to the plane of the page at the connections between crossed elements. For example, linear pantographs could be attached at points A and D or B and C in Figure 12. As the circle expands, the distance between these paired points decreases, which would force the linear pantographs and thus the entire structure to lengthen.

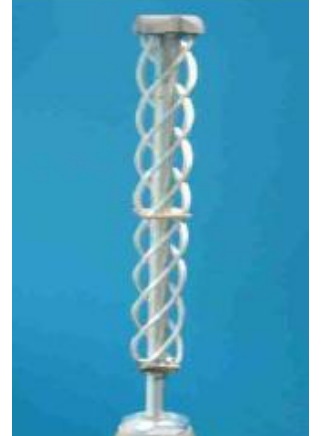

Figure 10. Quadrifilar helix antenna [18].
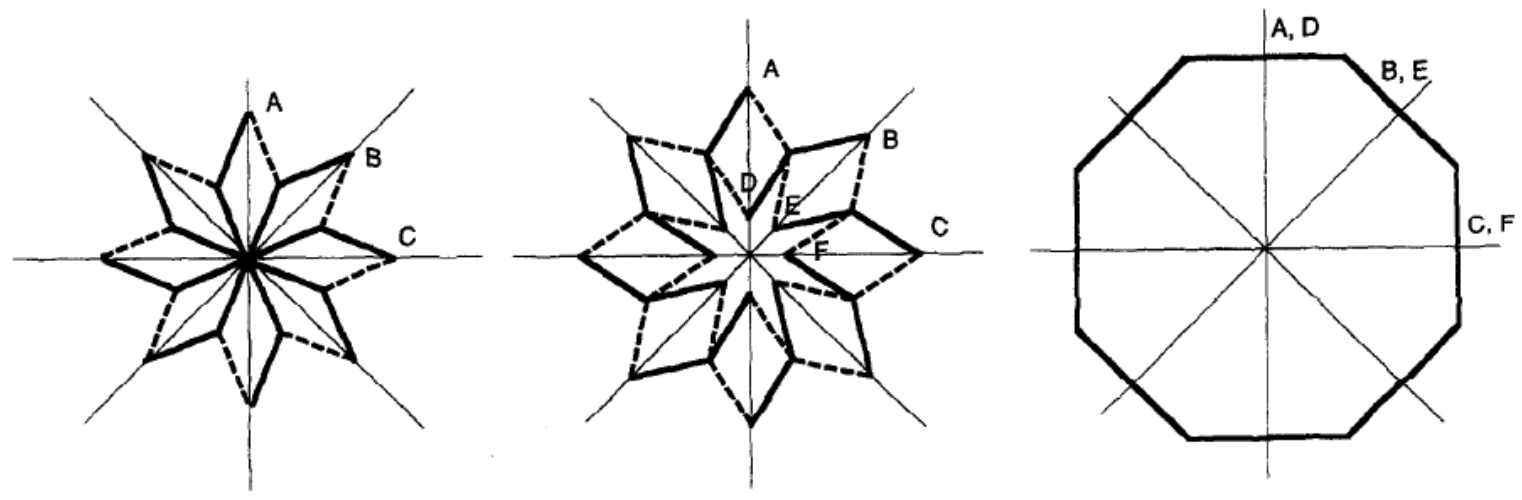

Figure 11. Expanding circular pantograph [19].

In this concept, the antenna conductors are thin rectangular elements attached to a cylindrical membrane. The membrane is attached to the structure but is not capped; that is, it does not have material covering the top and bottom circles.

The angle $\alpha$ is determined by the number of elements $n$ in the structure, using

$$
\alpha=\frac{360^{\circ}}{n}
$$

For the elements to form a mechanism the lengths $A F$ and $E F$ must satisfy (from [19])

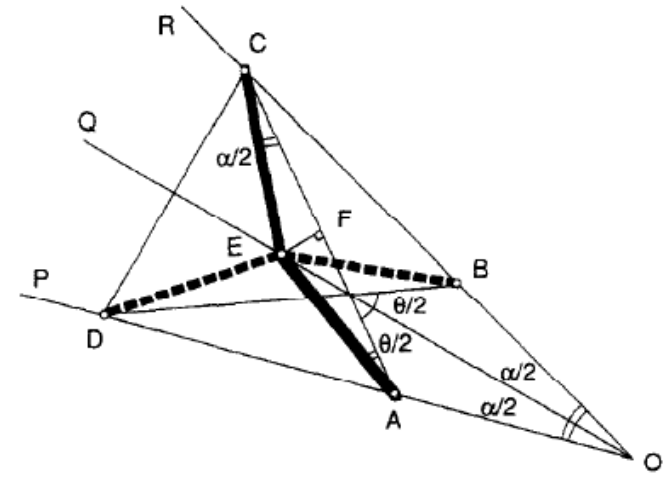

Figure 12. Angulated element [19].

$$
\tan \frac{\alpha}{2}=\frac{\overline{E F}}{\overline{A F}}
$$

The maximum achieved radius $R$ (measuring to the midpoint $F$ of the line $A C$ in each angulated element as seen in Figure 12), found using the right triangle AFO (note that this is only a right triangle in the entirely expanded configuration), is 


$$
R=\frac{\overline{A F}}{\tan \frac{\alpha}{2}}
$$

Finally, the desired height of the cylinder may be set by selecting the number of elements in each linear pantograph. Figure 13 demonstrates this concept in both the stowed and deployed configurations.
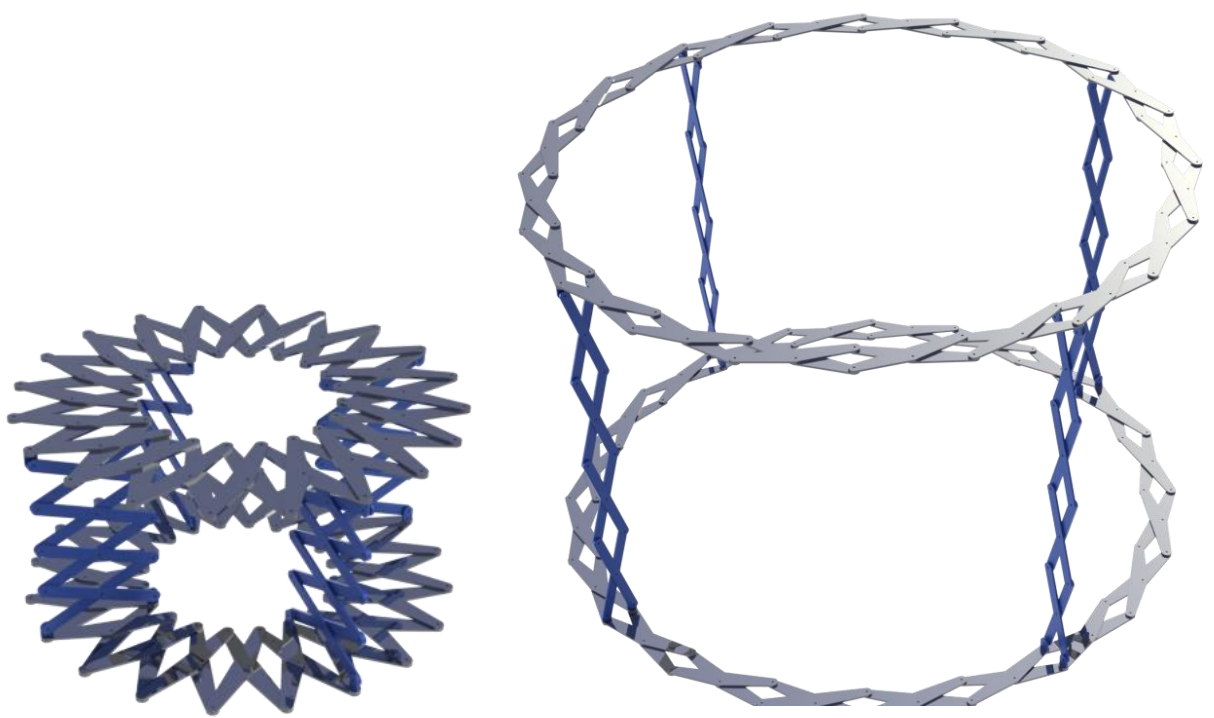

Figure 13. Cylindrical pantograph concept.

There are two distinct drawbacks to a pantograph design. First, it is mechanically complex, requiring many linkages and connections through which manufacturing error may be compounded. Second, the design does not miniaturize well because the minimum link and pivot size is limited by current fabrication processes. Assuming the angulated links may pass over each other, the minimum stowed diameter is $4 A F$ as shown by the leftmost image of Figure 11. This limitation suggests a compaction advantage to increasing the number of elements, which would decrease the length $A F$ without increasing the total diameter of the circular pantograph. It also suggests that the maximum length for the linear pantograph elements should be $2 A F$. Also worth note is that, of all the concepts presented, the cylindrical pantograph would likely be the most difficult to make self-deployable.

\section{Conclusion}

The goal of this work was to develop structural architecture concepts for deployable antennas that are efficient as both structures and antennas. The general approach has been to closely couple antenna theory and structural mechanics. Two types of structural architectures are proposed: shell structure and pantograph. Both architectures support antenna types that meet the stated requirements: circular polarization, wideband between 250 and $500 \mathrm{MHz}$, high gain, lightweight, structurally simple and able to reliably achieve the deployed configuration. The antenna types of interest include: crossed log periodic, conical log spiral, helical, and quadrifilar helix. Structural architectures that can be self deployed by stored elastic strain energy have been favored over those that require powered actuators or environmental effects to enforce deployment.

Designing antennas can be a highly non-linear affair. Small changes in geometry can yield large changes in antenna bandwidth, gain, and polarization. Widely variant antenna types can be engineered to meet a common set of antenna performance requirements. Therefore it is important for the structural designer to be involved early in the antenna design process.

Thus far, the concepts presented herein are relatively independent of conductor dimensions to allow maximum flexibility of design. In the future, structural requirements will be used to bound the possible frequencies each selected antenna is able to receive. For each design, material options will be explored and sizing and compaction equations will be expanded and refined. Finally, estimates of conductor dimensions will be refined to include the electromagnetic disturbance introduced by the structural elements. 


\section{References}

[1] Muri, P., Obulpathi, C., McNair, J., "Enhancing Small Satellite Communication Through Effective Antenna System Design," 2010 Military Communications Conference - Unclassified Program, San Jose, CA, November 2010.

[2] Ashida, H., "Design of Tokyo Tech Nano-Satellite Cute-1.7 + APD II and Its Operation," 59 $9^{\text {th }}$ International Astronautical Congress, Glasgow, Scotland, October 2008.

[3] Schaffner, J., "The Electronic System Design, Analysis, Integration and Construction of the Cal Poly State University CP1 CubeSat," $16^{\text {th }}$ AIAA/USU Conference on Small Satellites, Logan, UT, August 2002.

[4] Gao, S., "Antennas for Small Satellites," Loughborough Antennas and Propagation Conference, Loughborough, United Kingdom, March 2008.

[5] Fujishige, T., "Active Antennas for CubeSat Applications," $16^{\text {th }}$ AIAA/USU Conference on Small Satellites, Logan, UT, August 2002.

[6] Kitsuregawa, T., Advanced Technology in Satellite Communication Antennas, Artech House, Norwood, MA, 1990, p. 299.

[7] Kraus, J. Antennas, $2^{\text {nd }}$ ed,McGraw Hill, Inc, New York, 1988, p.270-271, 703-709.

[8] Freeland, R.E., Garcia, N.F. and Iwamoto, H., Wrap-rib antenna technology development, NASA Langley Research Center, p 139-166, N85-23813 14-15, 1984.

[9] Harless, R. I., Lightweight, compactly deployable support structure, Patent US 6,313,811 B1, 2001.

[10] Thomson, M.W., The AstroMesh deployable reflector, Antennas and Propagation Society International Symposium, IEEE, vol.3, pp.1516-1519, Aug 1999.

[11] de Selding, P. "Boeing Finishes Deployment of Stuck SkyTerra 1 Antenna," Space News, 14 December 2010. Accessed on

February 14, 2012 from <http://www.spacenews.com/satellite_telecom/101214-boeing-completes-skyterra-antennadeployment.html>.

[12] Charles, R. A., Design, Construction and testing of the deployable UHF antenna for Skynet 4 stage 2, IUTAM-IASS Symposium on Deployable Structures: Theory and Applications, Elsevier, 2000.

[13] Robinson, S., Simplified spacecraft antenna reflector for stowage in confined envelopes. Patent US 5,574,472, 1996.

[14] “3145BDP Dual-Polarized Log-Periodic Dipole Array,” ETS-Lindgren, <http://www.ets-lindgren.com/page/?i=3145BDP>, 22 July 2011.

[15] “3101 Conical Log Spiral Antenna,” ETS-Lindgren, <http://www.ets-lindgren.com/page/?i=3101>, 22 July 2011.

[16] Miura, K., "Method of Packaging and Deployment of Large Membranes in Space," The Institute of Space and Astronautical Science, Report No. 618, December 1985.

[17] "VHF and UHF Helical Antennas," WADE Antennas, Inc, <http://www.wade-antenna.com/TACO/Helicalantennas.pdf>, 23 July 2011.

[18] "S-Band Quadrifilar Helix Antenna," Surrey Space Centre,<http://www.sstl.co.uk/Downloads/Datasheets/Subsysdatasheets/S-Band-Quadrifilar-Helix-Antenna-ST0035522-v004-00>, 23 July 2011.

[19] You, Z., Pellegrino, S., "Foldable bar structures," International Journal of Solids and Structures, Vol. 34, No. 15, P. 18251847, May 1997. 